\title{
An Overview of 4G (4th Generation) Wireless Networks
}

\author{
Aiman J. Albarakati, \\ Department of Computer Engineering, \\ College of Computer and Information Science, \\ Majmaah University, \\ Majmaah, Saudi Arabia
}

\begin{abstract}
Generations of mobile and wireless communications has passed through an extensive broad and protracted way throughout diverse or unusual periods of progression as its nativity early on in the late 1970s. This sturdy and balanced comprehensive rumble in the population of mobile customers each and every year has intermittently rebuffed the growth of further and further refined technologies exhausting to hit the accurate harmony above all in provisions of stipulations of faultless worldwide roaming, quality and low-cost services, and towering data rate. At the moment abundant diverse technologies with their individual advantages and disadvantages are present worldwide. The approaching age of $4 \mathrm{G}$ systems is anticipating a prospective flat combination of all these diverse technologies with an accepted succession to hold up flawless economical high/towering data rate worldwide roaming, well-organized tailored services, distinctive user-centric incorporated service model, elevated Quality-of-Support and by and large unwavering system performance/recital. On the other hand, each stride in such scientific progressions brings massive research defies. In this research paper we will give you a detailed outline of $4 \mathrm{G}$ technology with its future prospects and research concerns/defies.
\end{abstract}

\section{Keywords}

Wireless communication, 4G, LTE

\section{INTRODUCTION}

The current epoch/age of smart and gifted wireless systems, successfully phrased as 4G (4th Generation) is leisurely enchanting profile to construct such decidedly intellectual, customer-based tailored service like virtual navigation realism or veracity. Unusual or diverse International engineering and research associations such as QualComm, NTT Nokia, DoCoMo, Ericsson, Alcatel, Motorola, WWRF, IEEE, ITU, 4GW-PCC, and Mobile VCE all were laid down to construct $4 \mathrm{G}$ wireless systems strike the mercantile marketplace. The changeover to data-oriented 2G (Second Generation) systems and digital voice in 1991 manifested the commencement of a multi-service platform/podium from the preceding monoservice age. Small data bit-rate and mono-media systems similar to TDM, GSM, IS-95 and cdmaOne, still exist in manifold worldwide positions. A provisional initiative and set up of $2.5 \mathrm{G}$ flanked by $2 \mathrm{G}$ and $3 \mathrm{G}$, endowed with an improved channel faculty/aptitude, elevated data rate and best performance and optimized transmission of packet-data progressing Internet entrée from unusual wireless devices. The profitable evolution to $3 \mathrm{G}$ (3rd Generation) systems in early 2002 scored the foundation of an actual multimedia age [1] of person-to-person prevailing interactivity. Core and central packet networked systems similar to cdma2000 and
WCDMA afford superior channel ability, 2 Mbps of a broadband data, towering speed multimedia transmission and worldwide roaming transversely a cellular/mobile network. This turned out to be the opening of jam-packed mammoth profits producing multimedia Internet applications and ecommerce (Electronic Commerce). Nevertheless, with the massive expansion in mobile customers worldwide each day and with up-and-coming hassles like fully user-centric services, elevated speed/pace streaming Internet multimedia services, faultless international roaming with ubiquitous(everpresent) coverage and unconstrained Quality-of-Service hold up. 3G systems have happened outlining their boundaries with spectrum provision, bandwidth accessibility, air intrusion standards and be short of seamless/flawless transport practices amid diverse networks. In addition, unusual undersized range communication systems like Bluetooth, WLAN and HIPERLAN plus broadcast communication systems with dissimilar characteristics crossed/spanned throughout this instance each with its individual virtues and demerits aiming special sorts of customers and unusual service types [2] fashioning the circumstances further intricate for $3 \mathrm{G}$ communications. These restrictions and negative aspects have engendered the prerequisite for a collective structure encircling all the offered heterogeneous/dissimilar wireless and wired systems in exercise. This IPv6-based prospective 4G structural design, usually depicted as MAGIC ((Mobile Multimedia, Anytime Anywhere Access) [3]. Comprehensive mobility hold incorporated wireless elucidation and tailored delicate service would be extremely vibrant and drastically grip the boundaries of $3 \mathrm{G}$ systems. Therefore, merged clarifications that can impeccably maneuver on the manifold, miscellaneous networks travel to the $4 \mathrm{G}$ setting gratifying the glut of next generation hallucination apparition on put into operation a translucent open wireless architecture (OWA), ought to be of the essence premeditated. This perceptibly encourages innovative defies on each stride and scientists wide-reaching countenance a rising charge of scheming proper elucidations.

\section{PROSPECTIVE RESEARCH DEFIES \\ 2.1 Mobile Terminals and Users}

Diverse manifold wireless and wired networks each one comprising its distinctive characteristics and endowing exclusive services make up the $4 \mathrm{G}$ setting. In support of wellorganized seamless/flawless worldwide/global roaming transversely the 4G OWA, clients or customers by means of multimode terminals ought to be routinely track and select a meticulous target or intended wireless system theoretically the most fitting one in a particular position putting forward the desirable service. Picking the preeminent fit network is mandatory to optimize the performance of system its QoS (Quality-of-Service), resources user-friendliness, outlays and 
service faculties/aptitude in that meticulous session [2][3]. Assume a customer stopovers by a shopping center where the WLAN or WLL is the wireless access network to get a hold a little vegetables automatically his re-configurable terminal tracks and connect/joins to the offered confined lofty speed wireless access system. Subsequently, when the user/client is driving a car athwart a main road, his similar terminal yet again selects and automatically switches to the proper wireless mobile network, e.g. CDMA2000 or GPRS. Nonetheless the selection method crystal clear to the end users in this whole network, the mobile terminal takes part in a considerable function. On behalf of the user predilections the mobile terminals algorithms perceive and decide on the most appropriate essential access network that endow with enhanced link layer connectivity at that fastidious position and re-configures itself according to the preferred network's requirements and set of laws. The meticulous facet of "on-thefly" (automatic network tracking and selection) is complex in $4 \mathrm{G}$ settings just because of its diverse temperament. In regards to this perspective, one of the projected ways out is "Access Network Selection" [3] where the multimode terminal accumulates satisfactory information for each meticulous communication session e.g. supported service types, customer subscription, outlays, Quality-of-Service, bandwidth necessities, pricing and coverage guidelines of the accessible core networks and accumulate them in the Service Platform (SP). The conclusion of which network to opt is based on the accumulated information in SP. Conversely restricted accessibility or non-accessibility and erroneousness in congregation the core network allied information are the most important hitches of this manner. Contrasting this practice where customer fondness on network assortment is discretionary, the [4] considerably mulls over user/customer preference/partiality even as deciding on a meticulous access network. [5] Illustrates a call admission policy-based method, which decides on the slightest encumbered accessible network in any finicky circumstances. Nevertheless this approach/method calls for further precise load balancing/harmonizing approach or practice. Despite all these research goings-on in this area to-date the most flourishing approach/method is the utilization of software radio (SR) devices that look into the on hand networks and later than downloading the suitable software(s) then re-configure themselves as accordingly the chosen network characteristics. Special downloading and reconfiguration approaches subsist and each one comprises its individual pros and cons. In [6] two approaches have been proposed are: (a) Reconfiguration over the air: In this a fanatical channel conduit between base station and user terminal smooth the progress of information swap over and fault-liberated downloading of the demanded software unit/module. Still this approach/method undergoes from the dilemma of managing the unreliable bandwidth rations in excess of narrow-band channels and as well of convincingly deal out a devoted concern physical channel for downloading. (b) Reconfiguration utilizing advanced SIm Cards: Here behalf on the preceding accumulated information concerning the re-configurable software modules/units on the smart cards (c) Internet Download: At this point software modules/units downloaded from Internet via modem, a terminal and personal computer are re-configured on the client's terminal. (d) Overthe-air (OTA) download [7] In this method multimode user terminals relentlessly keep an eye on the pre-defined dissemination comprehensive download channel (GPDCH), pilot and, on revealing of any accessible network, the terminals settle on whether to switch/swap over or not. Slow speed and slow downloading is the big issue here. In the $4 \mathrm{G}$ framework perspective Automatic network tracking and selection is a major research defy. The initiative of faultless worldwide roaming is greatly reliant on this. Desires are alive in crafting a unanimously acknowledged network tracking and selecting clarification or way out, which decide on most suitable available network in the light of most favorable resource practice, Quality-of-Service rations and customer inclinations. In view of this fact issues conferred at some point in the diverse downloading methods ought to be considered. On the other hand, it's an immense dispute or defies congregating satisfactory familiarity of each and every network prior to any selection is completed. Last but not least, prospect studies ought to concentrate on the predicament fields allied to find out the accessible access networks and their individual "points of presence" (PoPs), deciding on the suitable endorsement identifier for each PoPs and competently finding out the preeminent probable AAA routing and payload routing methods for flawless swap over of the payload packets amid the endpoints and substantiation/validation dialogue.

\subsection{Mobile Services}

In the age or epoch of 4G (4th Generation) it is decisive or of utmost importance to make certain that users/clients acquire expedient entrée to the services desirable at any agreed condition. From this perspective user/client mobility has turn out to be more significant facet in the devise of wireless communication systems of the next-generation. Researchers/Scientists have mulled over on three research areas in regards to such mobility concern. "Terminal mobility handles the mobility of users/clients comprising a solitary device". "Session mobility concerns with user in a PAN comprising manifold individual devices to endow with a live/subsist session and individual/personal mobility contemplates on stipulation of tailored in service settings for users all along with customer activities [2]. In personal/individual mobility a service desired by the client will be right away conveyed regardless of the client's position, device locality, operator/provider or supplier field and sort of network. Therefore, contrasting terminal mobility, personal and session mobility give attention to further on client activities as compared to terminal engagements. On placing of the cards on the terminals the configuration of the suitable services on the terminals come into existence. Still this maneuver is by and large appropriate for stand-alone terminals. Till date most of the research work has been carried out on personal mobility frameworks/structural designs for $4 \mathrm{G}$ systems. Regarding this we have two mobile agent-based personal mobility framework/structures. These agents intended to conduit the Internet and users. [9] Brings into play signaling protocols and mobile agents to assist large discretionary handling of the communication channels. This perks up the usability of the system and tolerates userfriendliness of mandatory services anytime worldwide. Nevertheless despite the fact that putting together personalized/tailored operating environment or settings and individual communication, this framework/structure undergoes from substantial outlays transparency. The [10] succeeding framework/structure make easy for the users/customers endowing backing in accessing emails, browsing, accessing files and in FTP(File transfer Protocol) correlated methods or practices. 
Equally session and personal mobility have strained some degree of research courtesy till this instant and to a great extent desirable to be made. The entire array of exertions carried out till now towards an inclusive way out flanked by the heterogeneous/diverse personality of the $4 \mathrm{G}$ systems and the session and personal mobility supervision concerns is thus far to pull off at all considerable shatter from first to last. Most important mystifications concerning the preference of SIP or Mobile IP (MIP) as the core/central protocol and whether the superlative structure ought to be based on Network Layer or either out to be an application Layer-based in case of personal and session mobility at a standstill keep it up with every one comprising its own merits and demerits. $4 \mathrm{G}$ endeavors at well-organized transmission of streaming data for video applications, which report for huge segment of prospect traffic, and calls for prevailing over the restrictions approximating high Qualityof-Service and bandwidth supplies, limited system resources, discrepancies in delays and lose of packets[11]. Services of satiated and streaming video are of two sorts of video services have putted on attractiveness in $4 \mathrm{G}$ systems. Nevertheless, whereas memory prerequisite for bursting is greatly outsized, streaming is deficient in usage or usability of bandwidth. So incorporation or realization of new-fangled applications of streaming video methods making best possible utilize of the existing bandwidth in restricted offered memory is the future delve into task. In this background selection of suitable protocols is vital. For video streaming TCP and UDP are the two central transport layer protocols. But UDP (User Datagram Protocol) undergoes from discriminating overcrowding interrelated concerns, which may possibly be more expediently gripped by the TCP (Transmission Control Protocol). From this perspective a well-organized method smoothing the progress of video streaming at extremely towering data rate has exposed that considerable expansion in cellular faculty and video superiority can be realized for [12]. Pull off elevated performance/recital grow is an imperative for streaming video applications. Bring into play of opportunistic scheduling practices confirmed to be a productive in this stance. [11] Reflects on equally channel burst and discrepancy of video traffic and illustrates that momentous performance/recital streaming video applications grow is at rest feasible. Incredibly lofty feature web service, reachable from side to side by bring into play the Java-based programs, are appropriate for the applications of e-commerce and mobile. Research carried out in this regards until now is insignificant and calls for considerable research concentration. To construct TCP a routine preference as the transport layer protocol over UDP, additional TCP friendly video streaming approaches over wireless networks are required for the reason that with improved streaming video applications all lay down to deluge the approaching wireless networks generation. UDP may possibly show the way to the unsteadiness of the Internet [12]. Good supervision of bandwidth and well-organized methods for endowing Quality-of-Service amid the endsystems are two extremely significant criterions for featured multimedia services. In this perspective problems of appropriate control of traffic, entrance control and Quality-ofSupport signaling methods entail further awareness.

\subsection{Wireless and Mobile Access Networks}

Customers/clients ought to be competent to roam generously and flawlessly athwart the geographic restrictions of unusual $4 \mathrm{G}$ networks upholding towering data rate, unsurpassed potential Quality-of-Service and acceptable connectivity with the application servers. Multimode terminals talented enough by design of tracking and selecting the apt offered fundamental network take part in an imperative task in this perspective. In this field generous research exploration have carried out with leading subjects in relation to dipping packet loss, signaling overhead and relinquish latency separately from raising the throughput. IPv6, endowing link-layer selfgoverning mobility administration elucidations, is collectively acknowledged as the principal backbone of the prospect $4 \mathrm{G}$ core/central network. Additional protocol progressions in this framework have put into practice Mobile IPv6 (MIPv6) and Hierarchical Mobile IPV6 (HMIPv6). Consequences of extra protocols at the unusual layers are also being calculated [13] in intensity. Sluggish grow in the number of successful Personal Area Networks (PANs) have recommended further research tasks in copping out dilemmas associated to mobility administration of roaming networks [14]. The progression of tracking and retaining the literal positions of wireless terminals by the primary system for promising connections when powered-on, powered-off or on the shift is position/location administration referred as a two phase method. Location tracking regards to the tracking with the accurate position of the terminals and location/position information storage [15] followed by keeping up further location information like Quality-of-Service qualifications/aptitude, traffic and validation. Roaming in the course of heterogeneous assortment of networks has specified ascend to the call for competent and incorporated location management techniques or methods. By now lots of successful location management approaches have been projected. [15] Has discussed an incorporated/integrated location management structural design for inter-network roaming. At this point the fundamental networks can bring together surrounded by themselves, track whether terminals are positioned inside the coverage area and therefore route inward packets/calls intended for individual/personal terminal. Nevertheless high reliability and ubiquitous coverage are the prime compensations at this point; thus far this method may perhaps endure from improved outlay of smart hardware and intricate programmable devices. Position of the coordinating servers in the original networks also carries out a vital role in managing the issues of location management. Projected location management structural's design may possibly be distributed, centralized, hybrid (combination of both) relying on whether all the underlying/fundamentals coordinating subnetworks are centrally supervised/handled or independently handled. Despite the fact that the centralized state of affairs gives the impression most favorable way other than it calls for a seal inter sub-network coordination and likelihood of signaling holdups are constantly present. The subsequent practice has the shortcoming of superior update outlay other than has greatly fewer paging overhead than the following one. Current studies has exposed that practically all the anticipated wireless mobility management Transport layer protocols have confirmed unsuccessful in managing dilemmas of location management. On the other hand, in [13] almost all higher layer protocols and Network layer props up wellorganized location management characteristics and may perhaps take part in prolific tasks in prospect studies on subjects similar to location assessment, location forecast and location seclusion. Further connected defies which call for awareness are stipulation of improved location coordination surrounded by the diverse assorted sub-networks, and 
optimally corresponding call to location update rate and mobility relation. Last but not least, particular consideration is mandatory about defies associated to handling the location of prospect mobile software modules/units that will take part in an imperative task in the subsequently generation network epoch/period. Resourceful handover management or handoff is an additional underlying subject of apprehension for keeping up worldwide mobility. Whereas roaming, flat handover of the mobile terminals is obligatory for flawlessly upholding the enduring communication. In this circumstance expansions in an assortment of intended protocols take part in a leading task. Despite the fact that MIPv6 was considered as the potential standard mobility protocol it undergoes from shortcomings approximating high packet failure, augmented system load, towering handover latency [2] and signaling scalability generally just because of nonappearance of hierarchy of location management, nonexistence of paging hold up and depletion of power of battery. Internet Engineering Task Force (IETF) has promoted HMIPV6 promoted as an auxiliary progression to MIPv6 has till date verified to be a talented/gifted technology for competently handling the MIPv6 shortcomings. Nevertheless, influences of HMIPv6 on $4 \mathrm{G}$ defense characteristics and roaming PANs have not been profoundly examined. Seamless or faultless handovers in a $4 \mathrm{G}$ State of affairs entails to grip defies coupled to equally inter-network inter-cellular (vertical), intra-network inter-cellular (horizontal) handover and [2] illustrates the perception of horizontal and vertical handover methods of a mobile terminal. Tribulations similar to stipulation of improved Quality-of-Support, dipping call droppings and disturbances, falling handover time, guaranteeing accurate handover achievement time, and best favorable successful call conclusion time are difficult to hold and all the IP-based sophisticated protocols ought to be systematically guarantees of congregating the defies. Worldwide roaming with multimode terminals is hold up by IPv6 for equally intra and inter technology handover methods. HMIPv6 also holds up both sorts of handovers. It trims down congestion and progress the handover pace [13]. [16] Is an innovative suggestion exerting to optimize MIPv6. It significantly lessens packet hammering and handover time in both sorts of handover. The other network, transport and advanced layer protocols successfully props up handover mechanisms/methods. Nonetheless, contrasting horizontal handovers, in business with vertical handover mechanisms/methods is greatly intricate. [5][17] Depicts successful handover management structural design of $4 \mathrm{G}$ vertical which lessens superfluous handover endeavors and abridges the signaling upshot. Enhanced capacity for additional considerable research in mounting an internationally accepted handover management structure for 4G systems is present. Despite the fact that numerous elucidations mingles Mobile IP with routing at an inferior layer are projected to considerably deal with handover allied complexity for dissimilar networks but overabundance of defies at a standstill continue living in all facets. Concerns linking to tumbling handover hindrances and Quality-ofService unpredictability in diverse systems call for the expansions of improved location ware adaptive application methods and precedence based algorithms. Other enduring research fields are to explore whether Virtual Connectivity Manager may possibly come out as an enhanced preference than Mobile IP in provisions of Internet mobility in prospect. On the other hand it would take significant amount of time prior to momentous handover handling framework/structure may possibly be profitably designed.

\subsection{Heterogeneous Networks Incorporation/integration \& Interoperability}

4th Generation systems are anticipated to smooth the progress of the flawless integration/incorporation and interoperation of an extensive assortment/series of offered systems like 3G systems, satellite broadband, fixed wireless access systems, WLL, PAN, WLAN, ad-hoc systems, WiMAX, WiBRO and the new-fangled beyond doubt broadband technologies with IP(Internet Protocol) as the infrastructure backbone. Unbolt wireless structural design be determined to endow with unconstrained connectivity, effusive broadband entrée, unhindered comprehensive roaming, worldwide Internet/ data/voice, ideal Quality-of-Service and client proscribed services is truly tricky to pull off owing to miscellaneous temperament of the access technologies in provisions of unreliable bit rates, bandwidth allotment, channel distinctiveness, fault-tolerance levels/extents and handoff management methods. [19] Demonstrates the feasible 4G interworking practice. An excess of worldwide broaden studies subsists scrutinized how and to what degree the faultless amalgamation of the diversified/broaden entrée networks ought to be passed on. The combined Ipv6-based obliging, diverse infrastructure would tolerate users/clients to roam worldwide with multimode terminals, accessing any service anyplace devoid of the Quality-of-Service getting influenced. [18] Illustrated three such likely combined structural designs. In the primary incorporated framework/structure multimode devices right to use the assorted services presented by the unusual fundamental networks. This architecture or structural designs endow with improved area coverage and call conclusion/completion other than non-economical. The succeeding incorporated/integrated framework is superimposed network-based where the diverse universal access points (UAPs) in the overlay/superimpose network decide on the offered fitting primary network for the customer. This structural design is multifaceted however props up improved subscription and billing. The subsequent architectural/structural design framework brings into play frequent access protocol and that's why other is less complex however is practicable barely in context of wireless ATMs. Researchers/scientists have discovered that the degree to which incorporation ought to be worked out relies on whether it is probable to put together the subsystems inside the offered standards or calls for further amendments. All-purpose tendency of incorporation and interoperation-oriented studies have acknowledged slack coupling, firm coupling and extremely rigid coupling as the three prospective incorporation or pairing/coupling methods for hot-spot expansions to $3 \mathrm{G}$ networks. Legitimate billing will be provided by loose coupling except underprivileged faultless connectivity and Quality-of-Service hold up, tight coupling prop up well-organized vertical handoffs nevertheless is thorny as well as impediment flat and such structural design can competently endow with radio resource measurement (RRM) and improved Quality-of-Service shore up. [20][24] Confers successful integration/incorporation approaches flanked by UMTS/GPRS/WWAN and WLAN every one with its individual merits and demerits mounting the by and large system performance/recital. [25] Proposed another multioperator proscribed, supple, multi-network 
integration/incorporation practice smoothing the progress of affluent end-to-end Quality-of-Service hold up. [26] Described an exclusive structure of TV broadcast and mobile cellular technologies in reality elevating the 4G Information civilization. Regardless of all these productive research attempts we at a standstill a protracted way to go prior to a collectively acknowledged, utterly translucent, user-focused, accommodating public-private wireless broadband communication framework or structure can be intended, which will be able to endow with faultless, ever-present coverage to the unrestricted network utilizing manifold diverse confidential systems [27]. This predicted IPv6 based absolutely packet-switched framework ought to be vigorously reconfigurable and layered plus animatedly self-governed and supportive in provisions of network maneuvers, organize, preservation and reprocess of self-governing modularized practical network blocks [28]. Massive research progressions are exerted in provisions of intending extremely developed integrated or incorporated Wireless Communication Systems [29].

\section{CONCLUSION}

The intuitive traits of $4 \mathrm{G}$ are beyond doubt stirring in the direction of getting unanimously acknowledged as the supreme communication system of next generation despite of having embarrassments of showing potential. $4 \mathrm{G}$ is envisaging as an assortment of diverse access technologies. In view of this fact, we outlined the feasible research defies under the unusual directions of users and mobile terminals, mobile services, wireless and mobile access networks, and communication defies/concerns. While some of other challenges/defies/issues have got much needy attraction and we also marked diverse acquainted or unacquainted research issues/defies, which call for considerable exertions for fulfilling $4 \mathrm{G}$ hallucinations.

\section{REFERENCES:}

[1] "B G Evans \& K Baughan", Visions of 4G, IEE Electronics and Communications engineering Journal, Autumn/Winter 2000.

[2] "S Y Hui \& K H Yeung", Challenges in the Migration to 4G Mobile Systems, IEEE Commuications, vol 41, no 12, Dec 2003.

[3] "R Eijk, J Brok, J Bemmel”, Access Network selection in a 4G Environment and the Roles of Terminal and Service Platform, Project: 4GPLUS, Wireless World Research Forum.

[4] [4] "M Calisti, T Lozza", An Agent- Based Middleware for Adaptive Roaming in Wireless Networks, Workshop on Agents for Ubiquitous Computing, AAMAS 2004, 20 July 2004, New York, USA.

[5] "K Murray, R Mathur", Network Access and Handover Control in Heterogeneous Wireless Networks for Smart Apace Environments, 1st International Workshop on Managing Ubiquitous Communications and Services (MUCS), Dec 11, 2003, Waterford, Ireland.

[6] "F Daneshgaran, M Laddamoda", On the Reconfigurability of a Software Radio Terminal for Supporting the Third and Fourth generation Wireless Standards, IEEE International Conference on Third Generation Wireless and Beyond, June 2001, San Francisco.
[7] "T H Le \& A H Aghvami”, Performance of an Accessing and Allocation Scheme for the Download Channel in Software Radio, Proc IEEE Wireless Commun and Net Conf, vol 2, pp 517-21, 2000.

[8] 'Network Discovery and Selection Problem"', InternetDraft of the Internet Engineering Task Force (IETF).

[9] "B Thai, R Wan", A Seneviratne \& T Rakotoarivelo, Integrated Personal Mobility Architecture: A Complete Personal Mobility Solution, Mobile Networks and Applications 8, pp 27-36, 2003.

[10] "A D Stefano \& C Santoro", NetChaser: Agent Support for Personal Mobility, IEEE Internet Comp, vol 4, no 2, Mar/April 2000, pp 74-79.

[11] "R S Tupelly, J Zhang", Opportunistic Scheduling for Streaming Video in Wireless Networks, 2003 Conference on Information Sciences and Systems, The Johns Hopkins University, March 12-14, 2003.

[12] "F H P Fitzek \& M Reisslein", Wireless video streaming with TCP and simultaneous MAC packet transmission (SMPT), International Journal of Communication Systems, vol 17, pp 421-435, 2004.

[13] "D Le, X Fu", A Review of Mobility Support Paradigms for the Internet, IEEE Communications Surveys \& Tutorials, 1st Quarter 2006, pp 38-51.

[14] F Paint, P Engelstad', , A M Nordvik, K Myksvoll \& S Svaet, Mobility Aspects in 4G Networks, Telenor 2002, White Paper.

[15] "U Varshney", Location management for wireless networks: issues and directions, Int $\mathrm{J}$ Mobile Communications, vol 1, no $1 / 2,2003$.

[16] "R Koodli", , Fast Handovers for Mobile IPv6, RFC 4068, July 2005.

[17] "W Zhang, J Jaehnert", , Design \& Evaluation of a Handover Decision Strategy for 4th Generation Mobile Networks, Proceedings of the 57th IEEE Vehicular Technology Conference, VTC 2003 Spring, Jeju, Korea, 2003.

[18] "U Varshney \& R Jain", Issues in Emerging 4G Wireless Networks, IEEE Computer, June 2001, vol 34, no 6, pp 94-96.

[19] “edro M Ruiz, Beyond” 3G: Fourth generation Wireless Networks, II Jornadas de Internet NG, Madrid, 22 October 2002.

[20] "J Chen, S H G Chan", , Mixed-Mode WLAN: The Integration of Ad Hoc Mode with Wireless LAN Infrastructure, IEEE Globecom 2003, 1-5 December, San Francisco, USA.

[21] "I S Misra \& A Banerjee", MPLS Based Mobility Framework in 4G Architectures, Proceedings of IEEE Tencon 2003, 15-17 October, Bangalore, India, 2003.

[22] "S L Tsao \& C C Lin", Design and evaluation of UMTSWLAN Interworking Strategies, Proceedings of Vehicular Technology Conference 2002, IEEE VTC 56th, Fall 2002

[23] "M Jaseemuddin", An Architecture for Integrating 
UMTS and 802.11 WLAN Networks, Proceedings of Eighth IEEE International Symposium on Computers and Communication ISCC03, 2003, p 716.

[24] "Flarion Technologies", Inc, 4 Ps for Carrier Employment of Mobile Broadband Systems, White Paper, Aug 2004.

[25] "N Niebert, A Schieder", , , Ambient Networks: An Architecture for Communication Networks beyond 3G, IEEE Wireless Communications, April 2004, vol 11, no 2, pp 14-22.

[26] "O Benali, K El-Khazen", , A Framework for an Evolutionary Path toward 4G by Means of Cooperation of Networks, IEEE. Communications Magazine, May 2004, pp 82-89.
[27] "K Ahmavaara, H Haverinen", Interworking Architecture Between 3GPP and WLAN Systems, IEEE Communications Magazine, vol 41, no 11, November 2003.

[28] "C Politis et al", Cooperative Networks for the Future Wireless World, IEEE Communications Magazine, vol 42, no 9, September 2004, pp 70-79.

[29] "S Zhou, M Zhao", , Distributed Wireless Communication System: A new Architecture for Future Public Wireless Access, IEEE Communications Magazine, March 2003, pp 108-113. 